\title{
Effect of Pollen-Specific Sublingual Immunotherapy on Oral Allergy Syndrome An Observational Study
}

\author{
Karl-Christian Bergmann, MD, * Hendrik Wolf, PhD, ${ }^{* *}$ and Jörg Schnitker, PhD
}

\begin{abstract}
Background: Oral allergy syndrome (OAS) triggered by fruit and vegetables often occurs in patients with pollen-induced rhinoconjunctivitis because of cross-reactive epitopes in pollen and associated foods. This open observational study examined the effect of pollenspecific sublingual immunotherapy ([SLIT] B. U. Pangramin or SLITone involving birch/alder/hazel, grasses/rye, and/or mugwort) on OAS triggered by several foods in patients treated in standard practice. Very few studies have examined SLIT use in this situation. Methods: Patients $(n=102)$ had pollen-induced rhinoconjunctivitis and OAS and were followed for up to 12 months. Baseline OAS (triggers, symptoms, and symptom severity) was assessed by questionnaire and patient history. Change in OAS was assessed using oral challenge test with 1 or 2 dominant food triggers (and compared with the sum score calculated from the OAS questionnaire at baseline) and clinician ratings of change. Pollen-induced rhinoconjunctivitis symptoms and medication use were also measured.

Results: In the oral challenge test, $77.0 \%$ of patients were considered responders (decrease in sum score of $\geq 50 \%$; no difference in patients receiving B. U. Pangramin or SLITone). At baseline, investigators rated OAS severity as at least moderate in $94.9 \%$ of patients compared with $36.9 \%$ after 12 months of treatment. After 12 months, OAS was rated as much or very much improved in $72.9 \%$ of patients. Sublingual immunotherapy significantly reduced rhinoconjunctivitis symptoms and medication use. Only $10 \%$ of patients experienced adverse drug reactions.

Conclusions: This study supplements the sparse literature on this topic and suggests that pollen-specific SLIT can reduce OAS triggered by pollen-associated foods in patients with pollen-induced rhinoconjunctivitis.
\end{abstract}

Key Words: immunotherapy, oral allergy syndrome, pollen, rhinoconjunctivitis, sublingual

(WAO Journal 2008;79-84)

Received for publication March 7, 2008; accepted March 7, 2008.

From the *Allergie-Centrum-Charité/ECARF, Department of Dermatology and Allergy, Charité-Universitätsmedizin Berlin, Berlin; **ALKSCHERAX Arzneimittel $\mathrm{GmbH}$, Wedel; and †Institut für angewandte Statistik GmbH, Bielefeld, Germany.

Supported by ALK-SCHERAX Arzneimittel GmbH, Wedel, Germany.

Reprints: Karl-Christian Bergmann, MD, Allergie-Centrum-Charité/ECARF, Department of Dermatology and Allergy, Charité-Universitätsmedizin Berlin, Luisenstraße 2-5, 10117 Berlin, Germany. E-mail: kcbergmann@, hotmail.com

Copyright (C) 2008 by World Allergy Organization
U $\mathrm{p}$ to $70 \%$ of patients with pollen allergy also experience oral reactions to food (oral allergy syndrome [OAS]), typically fruit and vegetables. ${ }^{1-3}$ Although most cases of OAS result in mild symptoms (itching of lips, mouth, and throat), more severe (lip and tongue swelling, angioedema of the pharyngeal mucosa) and even life-threatening (anaphylactic) symptoms can occur. ${ }^{4,5}$

Specific immunotherapy with pollen allergens may have a beneficial effect on pollen-associated food allergy triggering OAS because of cross-reactive epitopes present in pollen and associated foods. ${ }^{6,7}$ However, studies investigating the effect of subcutaneous pollen-specific immunotherapy on OAS report mixed results: some authors describe a reduction in OAS, whereas others have found no or only limited benefits from immunotherapy. ${ }^{8-14}$

Pollen-specific sublingual immunotherapy (SLIT) is an alternative way of administering specific immunotherapy, which has been shown to be effective in reducing polleninduced allergic rhinitis in several studies. ${ }^{15-19}$ Sublingual immunotherapy is applied directly to the oral mucosa, the site of the OAS reaction, and may therefore offer additional benefits over subcutaneous immunotherapy. This proposal is consistent with general clinical experience that initial mild adverse reactions to SLIT, such as oral itching and mucosal swelling, typically disappear during continued application of SLIT. $^{20}$

To our knowledge, only 2 studies have investigated the effects of pollen-specific SLIT on OAS triggered by pollen-associated foods. ${ }^{21,22}$ Hansen et $\mathrm{al}^{21}$ compared SLIT and subcutaneous immunotherapy with placebo in birch-allergic patients with OAS triggered by apple. No effect of immunotherapy on OAS was found, as assessed by a questionnaire and an open oral challenge test, but the number of patients receiving SLIT was small $(\mathrm{n}=11)$. Kinaciyan et $\mathrm{al}^{22}$ also failed to find an effect of birch-pollen SLIT on OAS to apple. Recently, however, Enrique et al ${ }^{23}$ showed that SLIT was effective in reducing food allergies: hazelnut-intolerant patients $(\mathrm{n}=12)$ demonstrated significant increases in tolerance to hazelnut after SLIT with hazelnut extract as assessed by a double-blind placebo-controlled food challenge in a randomized, double-blind, placebocontrolled study.

This open observational study further explores the influence of SLIT on OAS, thereby adding to the very limited literature in this area. The effects of immunotherapy with various pollen allergens in patients with pollen-induced rhinoconjunctivitis and pollen-associated OAS were recorded 
by practicing allergists during routine application of commercially available SLIT products.

\section{METHODS}

\section{Patients}

Patients had pollen-induced allergic rhinoconjunctivitis and concomitant OAS and did not have known contraindications to specific immunotherapy. Data were recorded between September 2002 and June 2005 during routine SLIT treatment by practicing allergists located throughout Germany.

\section{Study Design}

The study was open, noninterventional, and observational. Sublingual immunotherapy was administered by patients at home. Before SLIT initiation, demographic data, the history, and diagnostic measures with respect to pollen allergy and OAS were recorded. In an OAS questionnaire completed by the patients at that time, patients were asked to specify all foods that triggered their OAS symptoms, to name 1 or 2 foods provoking the most severe symptoms, to record the severity of symptoms (none/mild/moderate/severe) when last exposed to these foods, and to record the time of OAS onset after food intake. A weighted sum score was calculated from the 18 different symptoms assessed in this questionnaire (Table 1). Additional assessments (OAS severity, change in OAS status, response to foods) were performed during patient visits at the end of the initial treatment phase, and after 6 and 12 months of maintenance treatment (Table 2). The OAS severity and change in OAS status were assessed by the physician using the 7-point Clinical Global Impression Scales (OAS severity: not ill/borderline case/mildly ill/moderately ill/ markedly ill/severely ill/among the most extremely ill; change of OAS status: very much improved/much improved/only minimally improved/unchanged/minimally worse/much worse/very much worse). Response to foods was rated as: food avoided/food tolerated without reaction/food better

TABLE 1. The OAS Questionnaire and Weighted Sum Score

\begin{tabular}{rlc}
\hline No. & \multicolumn{1}{c}{ Symptom } & Weight \\
\hline 1 & Itchy lips & 1 \\
2 & Itchy tongue & 1 \\
3 & Itchy oral mucosa & 1 \\
4 & Burning tongue & 1 \\
5 & Swelling of the lips & 1 \\
6 & Swelling of the tongue & 1 \\
7 & Swelling of the oral mucosa & 1 \\
8 & Swelling of the larynx & 1 \\
9 & Inflammation of the tongue & 1 \\
10 & Inflammation of the oral mucosa & 1 \\
11 & Eye symptoms & 2 \\
12 & Nose symptoms & 2 \\
13 & Perioral skin symptoms & 2 \\
14 & Wheeze & 2 \\
15 & Dyspnea & 3 \\
16 & Nausea/vomiting & 3 \\
17 & Gastrointestinal disorders & 3 \\
18 & Collapse, shock & 4 \\
\hline
\end{tabular}

TABLE 2. Summary of Assessments During the Study

\begin{tabular}{|c|c|c|c|c|}
\hline & \multirow{2}{*}{$\begin{array}{l}\text { Study } \\
\text { Start }\end{array}$} & \multirow{2}{*}{$\begin{array}{c}\text { Initial } \\
\text { Treatment }\end{array}$} & \multicolumn{2}{|c|}{$\begin{array}{c}\text { Maintenance } \\
\text { Treatment }\end{array}$} \\
\hline & & & 6 Mo & $12 \mathrm{Mo}$ \\
\hline $\begin{array}{l}\text { Demographics and } \\
\text { history of pollen allergy }\end{array}$ & • & & & \\
\hline $\begin{array}{l}\text { History and current } \\
\text { diagnosis of OAS }\end{array}$ & - & & & \\
\hline OAS questionnaire & • & & & \\
\hline Severity of OAS & • & • & • & • \\
\hline $\begin{array}{l}\text { Course and global } \\
\text { improvement of OAS }\end{array}$ & & • & • & - \\
\hline $\begin{array}{l}\text { OAS challenge } \\
\text { test ( } 1 \text { or } 2 \text { times })\end{array}$ & & & • & • \\
\hline Rhinoconjunctivitis symptoms & $\bullet *$ & & $\bullet * *$ & $\bullet * *$ \\
\hline Rhinoconjunctivitis medication & $\bullet *$ & & $\bullet * *$ & $\bullet * *$ \\
\hline Nasal provocation test & - & & - & - \\
\hline SLIT, SLIT safety & & • & $\bullet$ & $\cdot$ \\
\hline Patients' diary & & & • & • \\
\hline
\end{tabular}

tolerated/reaction to food unchanged/food tolerance deteriorated. An open oral challenge test with the 1 or 2 foods identified as the most severe OAS triggers was scheduled after 6 and 12 months of maintenance therapy. The severity of symptoms triggered by the food challenge were assessed using a scale of none/mild/moderate/severe for 18 different symptoms and evaluated as weighted sum score as used for the OAS questionnaire (Table 1). Results from this test were compared with the weighted sum scores calculated from the OAS questionnaire at the beginning of SLIT.

$$
\text { Weighted sum score }=\sum_{\mathrm{i}=1}^{18} \text { weight }_{\mathrm{i}} \times \text { symptom }_{\mathrm{i}}
$$

A rhinoconjunctivitis symptom score from 0 to 21 was obtained by totaling scores for 3 eye symptoms (itching/tear flow/and redness) and 4 nasal symptoms (obstruction/itching/ rhinorrhea/sneezing): each symptom was scored retrospectively for the preceding pollen season on a scale of 0 to 3 (no/ mild/moderate/severe symptoms). Lung symptoms were assessed for 4 items (chest tightness/wheezing/cough/dyspnea), and skin symptoms, for 2 items (pruritus/eczema). Optionally, a nasal provocation test (NPT) was performed according to the Position Paper of the German Society for Allergology and Clinical Immunology about application of the NPT on diseases of the upper airways. ${ }^{24}$

Patients were also asked about medication use (Yes/No for a range of typical allergy medications) and whether sleeping was impaired by their allergy symptoms. Rhinoconjunctivitis symptoms were reassessed during the first pollen season after starting SLIT, and the NPT was optionally performed. At the end of the 12-month observation period, the status of the patient in the first pollen season with SLIT compared with the pretreatment season was assessed globally by patient and physician (better/unchanged/worse/not assessable). The doses applied and potential adverse reactions throughout the study were recorded in patient diaries. 


\section{SLIT Preparations and Dosage}

Sublingual immunotherapy was typically initiated with B. U. Pangramin SLIT pollen allergens [grass mix/rye, tree mix (hazel/alder/birch) and mugwort; ALK-SCHERAX Arzneimittel GmbH, Wedel, Germany], although a few patients started on SLITone (available from November 2003; ALK-SCHERAX Arzneimittel GmbH). The recommended treatment regimen for B. U. Pangramin SLIT involved an initial 4-week updosing phase followed by maintenance treatment involving 10 drops from a vial of strength 500 Specific Treatment Units Abello (STU)/mL 3 times a week. For SLITone, the recommended regimen involved an initial 10-day updosing phase followed by a single daily maintenance dose of $200 \mathrm{STU}$ (0.2 mL of $1000 \mathrm{STU} / \mathrm{mL}$ concentration). During the updosing phases, patients were advised to increase the dose according to the dosage schedule only if the previous dose had been applied and if the dose was well tolerated. If adverse reactions occurred, patients were instructed to contact their physician. For both products, patients were told to keep the SLIT drops under the tongue for 2 to 3 minutes before swallowing. During the study, some patients switched from B. U. Pangramin SLIT to SLITone grass mix and rye or tree mix. In these cases, treatment with SLITone was always started with the initial 10-day updosing phase.

\section{RESULTS}

\section{Patient Characteristics}

Overall, 102 patients were treated with at least 1 dose of SLIT (88 patients were treated with B. U. Pangramin SLIT, 11 were initially treated with B. U. Pangramin and then transferred to SLITone; 3 patients initiated SLIT with SLITone). Seventy-seven patients were considered to have completed the 12-month observation period. Reasons for not completing the study were adverse events $(\mathrm{n}=8)$, lack of compliance (patients, $n=6$; investigators, $n=3)$, improvement $(n=3)$, lack of efficacy $(n=2)$, lack of compliance + lack of efficacy $(\mathrm{n}=1)$, termination by the patient $(\mathrm{n}=1)$, and desire to start a family $(n=1)$; data from 2 of these patients were, however, included in some of the analyses. Of the 99 patients receiving B. U. Pangramin SLIT, 87 achieved the maximum maintenance dose after 4 weeks $(n=75)$ or delayed after a longer updosing phase $(\mathrm{n}=12)$. Three patients were lost to follow-up at an unknown maintenance dose, 4 discontinued treatment at a submaximum level because of allergic adverse reactions, 3 patients named allergic symptoms because of pollen exposure as the reason for discontinuing updosing (no subsequent continuation was documented), and in 2 patients, a submaximum maintenance dose was chosen because of other medical reasons. In total, the maximum B. U. Pangramin SLIT dosage was reached in $87.9 \%$ of the patients.

Patient characteristics and OAS history at baseline are shown in Table 3. Patients were most frequently sensitized to birch/alder/hazel followed by grasses/rye and mugwort. Accordingly, patients experienced OAS reactions predominantly to tree-associated foods: apple $(81.8 \%)$ and hazelnut (73.7\%), and with lower frequency to carrot (27.3\%). Patients completing the OAS questionnaire named 175 dominant

\begin{tabular}{lc}
\hline TABLE 3. Patient Characteristics and OAS History & at Baseline \\
\hline Patients Treated With SLIT & $\mathbf{n}=\mathbf{1 0 2}$ \\
\hline Median age, yrs (range) & $34.5(6-64)$ \\
Children $<14$ yrs, \% & 13.7 \\
Male/female, \% & $33.3 / 66.7$ \\
Median duration of rhinoconjunctivitis, yrs (range) & $8.0(1-37)$ \\
Patients with pollen-induced airway & 62.7 \\
symptoms, \% & \\
Patients with pollen-induced skin & 23.5 \\
symptoms, \% & \\
Positive skin prick test to: & $\mathrm{n}(\%)$ \\
Grasses and rye & $63(67.7)$ \\
Birch, alder, hazel & $93(93.9)$ \\
Beech, oak, ash & $35(50.7)$ \\
Mugwort & $26(34.2)$ \\
Other pollen & $5(12.8)$ \\
House dust mites & $27(34.2)$ \\
Animal dander & $22(31.0)$ \\
SLIT treatment with (includes multiple entries): & $\mathrm{n}(\%)$ \\
Tree mix (birch, alder, hazel) & $81(79.4)$ \\
Grasses and rye & $38(37.3)$ \\
Mugwort & $6(5.9)$ \\
OAS history & \\
Patients evaluable & $\mathrm{n}=99$ \\
Median duration of OAS, yrs (range) & $5.0(0-23)$ \\
Predominant triggers, \% & \\
Apple & 81.8 \\
Hazelnut & 73.7 \\
Carrot & 27.3 \\
Predominant symptoms & 32.6 \\
Itchy lips, tongue, and/or oral mucosa & \\
Swelling of lips, tongue, oral mucosa, and/or larynx & 74.3 \\
Burning of tongue & \\
Eye symptoms & \\
Nose symptoms & \\
\hline
\end{tabular}

triggers as causing the most marked symptoms. The most commonly named dominant triggers in the questionnaire were apple $(33.1 \%, 58 / 175)$, hazelnut $(22.3 \%, 39 / 175)$, carrot $(8.0 \%, 14 / 175)$, peach $(3.4 \%, 6 / 175)$, and celery $(3.4 \%, 6 /$ 175). Symptoms to these dominant triggers mostly occurred within 5 minutes of eating the food, and $25.4 \%$ of symptoms were considered severe. Only $7.1 \%$ of reactions required immediate medical attention. Most patients (79.4\%) received SLIT with tree allergens, $37.3 \%$ received SLIT with grasses and rye, and $5.9 \%$ received SLIT with mugwort.

\section{Effect of SLIT With Pollen Allergens on OAS}

During the course of the study, patients frequently avoided foods that provoked OAS reactions. The rate of food avoidance for dominant OAS triggers decreased from $65.5 \%$ after 4 weeks' treatment to $40.0 \%$ after 12 months (Fig. 1); 71 $(40.6 \%)$ of the 175 named dominant triggers of OAS were avoided during the study. After 4 weeks' treatment, 24 (40.7\% of patient cases) of the 59 food types ingested were tolerated without reaction or better than previously. After 12 months, this value increased to $86.4 \%(70 / 81)$. 

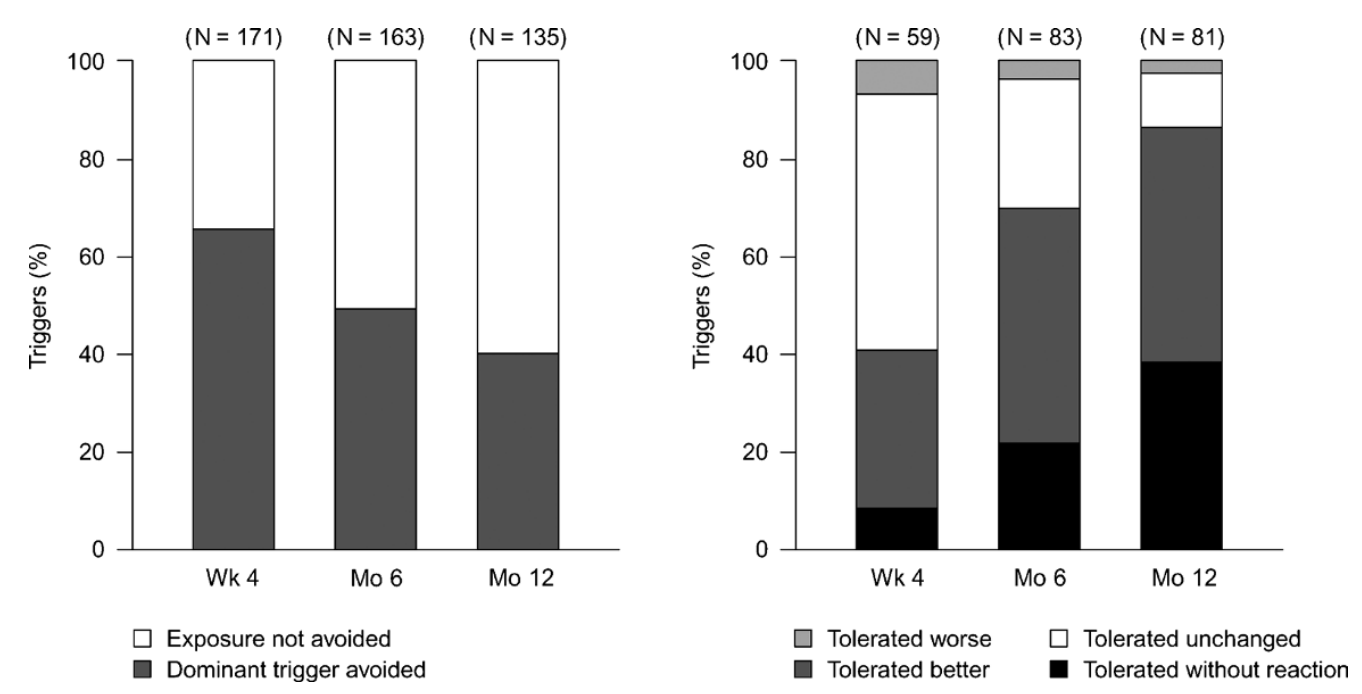

FIGURE 1. Exposure to, and tolerability of, dominant OAS triggers.

In the oral challenge test, weighted sum score decreased by a mean of 64.3 relative to the reactions recorded in the OAS questionnaire before SLIT initiation. Defining a response as a decrease in sum score of $50 \%$ or more, $77.0 \%$ of patients were responders in the oral challenge test (confidence interval, 64.5-86.9, $P<0.0001, \mathrm{n}=61$ ), and most of these patients experienced a reduction in sum score of more than $75 \%$ (Fig. 2). There was no significant difference in response in patients receiving B. U. Pangramin SLIT or SLITone. In patients with apple intolerance (the most common food trigger) who underwent an oral challenge test with this food, $89.2 \%$ (33/37) responded to SLIT (decrease in sum score of at least $50 \%$ ). The respective percentage for hazelnutintolerant patients was $69.6 \%(16 / 23)$.

Investigators reported a decrease in OAS severity after SLIT; at baseline, $94.9 \%$ of patients were rated as at least moderately ill compared with $36.9 \%$ at month 12 . Furthermore, after 6 months of SLIT treatment, investigators rated OAS as much or very much improved in $53.9 \%$ (48/89) of patients. This percentage increased to $72.9 \%(54 / 74)$ in patients completing 12 months of therapy. In all patients receiving treatment (regardless of how long), 65.7\% (65/99) were rated by the investigators as much or very much improved.

\section{Effect of SLIT on Pollen-Induced Rhinoconjunctivitis}

Sublingual immunotherapy treatment markedly decreased rhinoconjunctivitis symptoms during the pollen season in the completer population (Fig. 3). The mean symptom severity score for eye symptoms decreased by $58.4 \%$, nose symptoms by

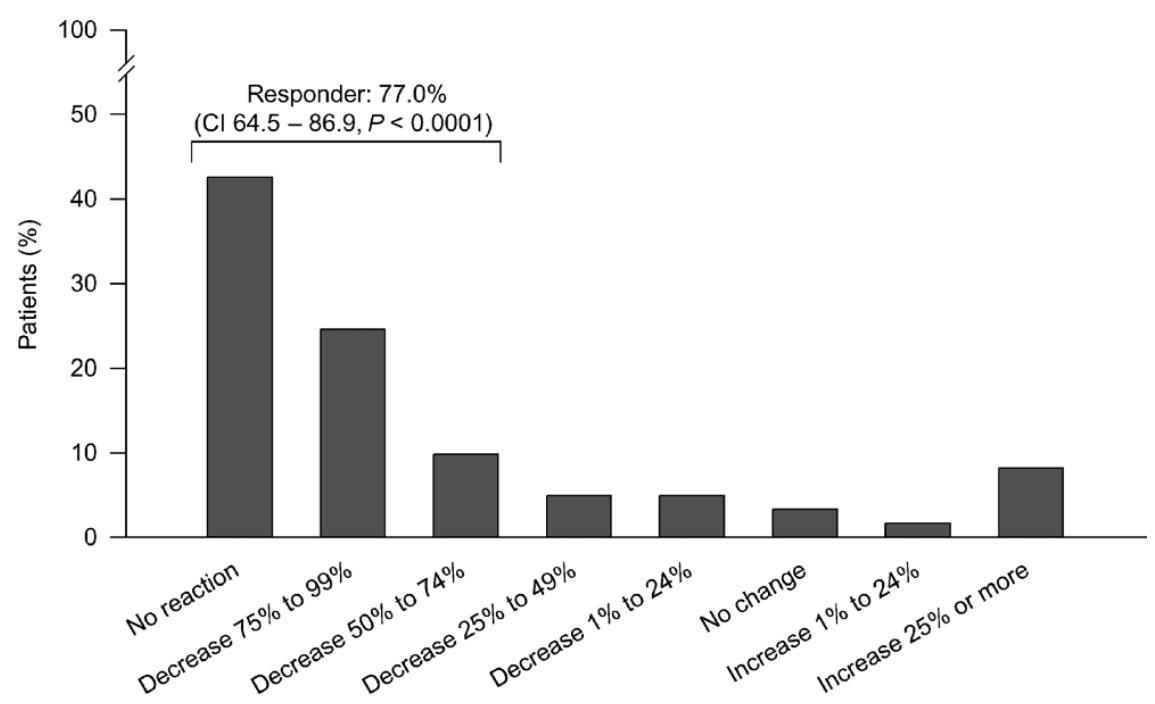

FIGURE 2. The OAS challenge test at 6 and 12 months compared with the results of the OAS questionnaire at baseline (weighted sum score). The figure presents individually the last findings for each included patient ( $n=61 ; 8$ tests at 6 months, 53 tests at 12 months). $\mathrm{Cl}$ indicates confidence interval. 


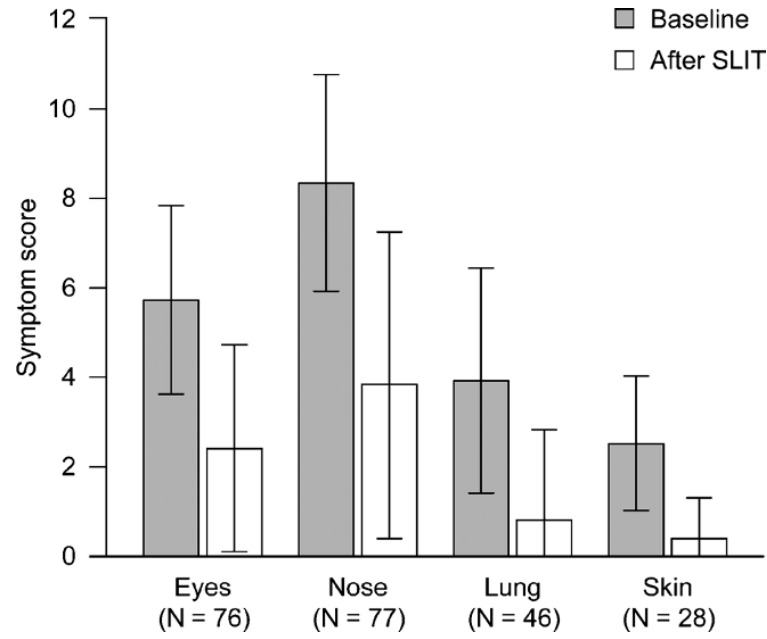

FIGURE 3. Course of rhinoconjunctivitis symptom scores.

$54.2 \%$, bronchial symptoms by $79.2 \%$, and skin symptoms by $85.9 \%$. Insomnia improved in $60.0 \%$ of cases.

After SLIT, symptomatic medication was reduced or no longer required in $92.1 \%(\mathrm{n}=70)$ of patients who were using medication at baseline $(n=76)$. Forty-nine patients completed an NPT before and after SLIT; $34.7 \%$ of them had a positive test before and a negative test after SLIT. Of patients and investigators, $84.2 \%$ rated well-being during the pollen season as improved after SLIT relative to the last pollen season before immunotherapy. There was no difference in well-being assessment between B. U. Pangramin and SLITone.

\section{Tolerability of SLIT}

Of the 99 patients who received at least 1 dose of $B$. U. Pangramin SLIT, 10 (10.1\%) had a total of 14 adverse drug reactions [asthma attack $(n=2)$, nausea $(n=2)$, retching, neurodermatitis, angina, diarrhea, OAS worsening, anaphylactic event of undetermined origin, prickling of the tongue $(\mathrm{n}=2)$, numbness of the tongue, burning of the tongue]. No adverse drug reactions were recorded with SLITone. Only 1 patient discontinued SLIT because of deterioration of OAS after the initial therapy. In total, 8 of the adverse drug reactions resulted in treatment discontinuation. These included asthma $(n=2)$ and single cases of OAS worsening, severe angina, neurodermatitis, nausea, anaphylactic event of undetermined origin (not temporally related to SLIT), and prickling tongue. Overall, $94.7 \%$ of patients and $95.7 \%$ of physicians rated the tolerability of initial SLIT as very good (patients, $76.8 \%$; physicians, $77.7 \%$ ) or good (patients, $17.9 \%$; physicians, $18.1 \%$ ). Similar results were reported after 6 and 12 months of maintenance therapy (patients, 93.9\%; physicians, 96.3\%; and patients, 94.7\%; physicians $96.1 \%$, respectively).

\section{DISCUSSION}

In this open observational study, we found that patients treated with pollen-specific SLIT during routine clinical treatment showed improvements in OAS triggered by various pollen-associated food allergens (when these foods were ingested naturally or during an oral challenge test) and in pollen-induced rhinoconjunctivitis. Furthermore, the improve- ment increased as treatment continued for up to 12 months. Physicians rated OAS as much or very much improved in nearly $75 \%$ of patients after 12 months of treatment, and $77 \%$ of patients had a favorable response in the open oral challenge test. The symptoms of allergic rhinoconjunctivitis were also improved during the first pollen season after SLIT treatment.

Although our study was not controlled, OAS does not normally improve without treatment. ${ }^{2,8,11}$ For example, in a study of specific injection immunotherapy in birch pollensensitive patients with apple-induced OAS, none of the 20 control patients reported a reduction in symptoms during a 12 - to 48 -month follow-up period ${ }^{8}$; in contrast, $84 \%$ of immunotherapy-treated patients reported complete disappearance or significant improvement in symptoms. It is our opinion that an improvement in OAS symptoms to the extent reported in this study would not normally be expected without treatment. Furthermore, annual variations in pollen count are unlikely to account for our findings because patients were recruited and treated over several years and were allergic to different pollens.

To our knowledge, only 2 other studies ${ }^{21,22}$ have investigated the effect of pollen-specific SLIT in patients with concomitant OAS. In both studies, no effect of birchallergen SLIT or subcutaneous immunotherapy on OAS triggered by apple was reported relative to placebo, but the number of patients treated with SLIT and assessed by food challenge was low (11 patients ${ }^{21}$ and 9 patients $\left.^{22}\right)$. The recently published study by Kinaciyan ${ }^{22}$ classified patients allergic to birch who had been treated with SLIT as responders and nonresponders according to the outcome in an NPT. The 9 patients who had a positive response in the NPT were tested by double-blind placebo-controlled food challenge and oral food challenge with apple. No significant differences between the food challenges at baseline and after 1 year of SLIT were observed. The authors speculate based on the results of T-cell proliferation experiments that an exclusively food-specific T-cell response to Mal d 1 (a protein with an amino acid sequence that is $64 \%$ similar to the major birch pollen allergen, Bet v 1) may exist that can only be altered if OAS-related food allergens are contained in the vaccine for SLIT. Consistent with this argument, another study showed that patients with OAS and food allergy to hazelnut can be successfully treated with SLIT using a hazelnut extract containing the most relevant allergens triggering OAS and food allergy. 23

Nevertheless, our observational study involving a much larger sample of 102 patients with pollen-induced allergy and concomitant OAS suggests that patients treated routinely with pollen allergen SLIT can report positive effects of SLIT on OAS. This is consistent with several studies that have shown benefits of subcutaneous pollen-specific immunotherapy on concurrent OAS using open ${ }^{2,8,13,14}$ or blinded, ${ }^{11}$ and controlled $^{2,8,11,14}$ or uncontrolled ${ }^{13}$ designs. Furthermore, SLIT may have the potential to influence OAS more strongly than subcutaneous treatment because of the application of allergens directly to the oral mucosa and the subsequent induction of oral tolerance. To explore the effect of SLIT on OAS further, controlled blinded clinical trials should be undertaken. Nevertheless, this study extends the available literature in 
this field, particularly by studying a wider variety of patients, treatments, and OAS triggers, more indicative of the real-life clinical setting.

\section{ACKNOWLEDGMENT}

The authors thank Dr. J. Oswald for his assistance.

\section{REFERENCES}

1. Eriksson NE, Formgren H, Svenonius E. Food hypersensitivity in patients with pollen allergy. Allergy 1982;37:437-443.

2. Bucher X, Pichler WJ, Dahinden CA, Helbling A. Effect of tree pollen specific, subcutaneous immunotherapy on the oral allergy syndrome to apple and hazelnut. Allergy 2004;59:1272-1276.

3. Yamamoto T, Asakura K, Shirasaki H, Himi T, Ogasawara H, Narita S, Kataura A. Relationship between pollen allergy and oral allergy syndrome [in Japanese]. Nippon Jibiinkoka Gakkai Kaiho 2005;108:971-979.

4. Mari A, Ballmer-Weber BK, Vieths S. The oral allergy syndrome: improved diagnostic and treatment methods. Curr Opin Allergy Clin Immunol 2005;5:267-273.

5. Sloane D, Sheffer A. Oral allergy syndrome. Allergy Asthma Proc 2001;22:321-325.

6. Ebner C, Hirschwehr R, Bauer L, Breiteneder H, Valenta R, et al. Identification of allergens in fruits and vegetables: IgE cross-reactivities with the important birch pollen allergens Bet $\mathrm{v} 1$ and Bet $\mathrm{v} 2$ (birch profilin). J Allergy Clin Immunol 1995;95:962-969.

7. Vanek-Krebitz M, Hoffmann-Sommergruber K, Laimer da Camara Machado M, Susani M, Ebner C, et al. Cloning and sequencing of Mal d 1 , the major allergen from apple (Malus domestica), and its immunological relationship to Bet $\mathrm{v} 1$, the major birch pollen allergen. Biochem Biophys Res Commun 1995;214:538-551.

8. Asero R. Effects of birch pollen-specific immunotherapy on apple allergy in birch pollen-hypersensitive patients. Clin Exp Allergy 1998;28:1368-1373.

9. Asero R. How long does the effect of birch pollen injection SIT on apple allergy last? Allergy 2003;58:435-438.

10. Asero R. Effects of birch pollen SIT on apple allergy - a matter of dosage? Allergy 2004;59:1269-1271.

11. Bolhaar ST, Tiemessen MM, Zuidmeer L, van Leeuwen A, Hoffmann-Sommergruber K, et al. Efficacy of birch-pollen immunotherapy on cross-reactive food allergy confirmed by skin tests and double-blind food challenges. Clin Exp Allergy 2004;34:761-769.

12. Kelso JM, Jones RT, Tellez R, Yunginger JW. Oral allergy syndrome successfully treated with pollen immunotherapy. Ann Allergy Asthma Immunol 1995;74:391-396.

13. Herrmann D, Henzgen M, Frank E, Rudeschko O, Jäger L. Effect of hyposensitization for tree pollinosis on associated apple allergy. J Investig Allergol Clin Immunol 1995;5:259-267.

14. Modrzynski M, Zawisza E, Rapiejko P, Przybylski G. Specific-pollen immunotherapy in the treatment of oral allergy syndrome in patients with tree pollen hypersensitivity [in Polish]. Przegl Lek 2002;59: 1007-1010.

15. Passalacqua G, Lombardi C, Canonica GW. Sublingual immunotherapy: an update. Curr Opin Allergy Clin Immunol 2004;4:31-36.

16. Marogna M, Spadolini I, Massolo A, Canonica GW, Passalacqua G. Randomized controlled open label study of specific immunotherapy for respiratory allergy in real life: clinical efficacy and more. Allergy 2004;59:1205-1210.

17. Wilson DR, Torres LI, Durham SR. Sublingual immunotherapy for allergic rhinitis. Cochrane Database Syst Rev 2003;(2):CD002893.

18. D’Ambrosio FP, Ricciardi L, Isola S, Savi E, Parmiani S, Puccinelli P, Musarra A. Rush sublingual immunotherapy in Parietaria allergic patients. Allergol Immunopathol (Madr) 1996;24:146-151.

19. Feliziani V, Lattuada G, Parmiani S, Dall'Aglio PP. Safety and efficacy of sublingual rush immunotherapy with grass allergen extracts. A double blind study. Allergol Immunopathol (Madr) 1995;23:224-230.

20. Di Rienzo V, Pagani A, Parmiani S, Passalacqua G, Canonica GW. Post-marketing surveillance study on the safety of sublingual immunotherapy in pediatric patients. Allergy 1999:54:1110-1113.

21. Hansen KS, Khinchi MS, Skov PS, Bindslev-Jensen C, Poulsen LK, Malling HJ. Food allergy to apple and specific immunotherapy with birch pollen. Mol Nutr Food Res 2004;48:441-448.

22. Kinaciyan T, Jahn-Schmid B, Radakovics A, Zwölfer B, Schreiber C, et al. Successful sublingual immunotherapy with birch pollen has limited effects on concomitant food allergy to apple and the immune response to Bet v 1homolog Mal d 1. J Allergy Clin Immunol 2007;119:937-943.

23. Enrique E, Pineda F, Malek T, Bartra J, Basagana M, et al. Sublingual immunotherapy for hazelnut food allergy: a randomized, double-blind, placebo-controlled study with a standardized hazelnut extract. J Allergy Clin Immunol 2005;116:1073-1079.

24. Riechelmann H, Bachert C, Goldschmidt O, Hauswald B, Klimek L, Schlenter WW, et al. German Society for Allergology and Clinical Immunology (ENT Section); Working Team for Clinical Immunology. Application of the nasal provocation test on diseases of the upper airways. Position paper of the German Society for Allergology and Clinical Immunology (ENT Section) in cooperation with the Working Team for Clinical Immunology [in German]. Laryngorhinootologie 2003;82:183-188. 